Rakenteiden Mekaniikka (Journal of Structural Mechanics)

Vol. 50, No 3, 2017, pp. 256-260

https://rakenteidenmekaniikka.journal.fi/index

https:/doi.org/10.23998/rm.65075

(c)Author(s) 2017.

Open access under CC BY-SA 4.0 license.

\title{
Microstructural modelling of materials
}

Merja Sippola ${ }^{1}$, Anssi Laukkanen, Tom Andersson, Matti Lindroos

Summary. This extended abstract summaries the VTT ProperTune ${ }^{\mathrm{TM}}$ materials modelling approach.

Key words: materials, modelling, finite element method, image based model, synthetic model

Received 26 June 2017. Accepted 12 August 2017. Published online 21 August 2017.

\section{Characterisation and image based modelling}

Modern characterisation methods like the Scanning Electron Microscope (SEM) especially equipped with an Electron Backscatter Diffraction (EBSD) detector, X-ray tomography and Transmission Electron Microscopy (TEM) have enabled much more reliable modelling of material microstructures, even non-periodic and porous ones, than what was possible with simple geometric primitives. The SEM and TEM typically create 2D images of material surfaces at small size scales. With the EBSD it is possible to obtain accurate crystallographic information of different materials. With X-ray tomography it is possible to take 3D images of material microstructures at relevant size scales.

With modern image analysis tools one can process and segment the image and divide it into separate phases of the microstructure, for examples wood and polymer in a Wood-Polymer Composite (WPC). See Figure 1. With the image based meshing tools these images can be meshed with solid elements to create finite element models. Meshing tools also allow generation of node sets for loads and boundary conditions and contact surfaces to be used in a Finite Element (FE) code like ABAQUS, as well as material definitions and section definitions to attach the material definitions to the element sets. Then the model can be imported into a FE program, create the loadings and boundary conditions and contact definitions, and finally run the FE model and study

\footnotetext{
${ }^{1}$ Corresponding author. merja.sippola@vtt.fi
} 
the results visually and numerically. Much in these programs can also be tailored and workflows automated using Python and like scripting languages.

\section{Challenges in image based modelling}

Some of the challenges in image based modelling are related to image quality and segmentation. The segmentation algorithms are mostly tackling the $2 \mathrm{D}$ slices of the tomography image instead of 'seeing in 3D'. Besides this the image resolution and quality are not always perfect, and the greyscale difference between material phases can be small. As a result it is in for example a WPC difficult to avoid artificial joining of the fibers, which would result in too stiff behavior for the composite. Careful segmentation by a skilled expert is needed. The alternative is to cut these artificial links afterwards by a Python script.

Another problem in image based modelling is that it often creates very large models, as the images contain lots of small details (holes, islands, surface wrinkles etc). Careful processing and simplification of the image in the segmentation phase is needed. It is often important to remove very small objects that would not have much effect on the mechanical behavior but would result in very small elements. This is called island removal. Alternatively the minimum element size in meshing may be set large enough to avoid too small elements. A tougher problem is that the resulting model of for examples a porous foam material may contain separate parts that in the FE model would move as individual rigid bodies, and also parts that are attached to other parts in the model by only one or two nodes (a hinge). There are scripts and routines to clean the model of small hinged parts and to reveal and attach the separate parts. The size of the modelled volume, often called a Representative Volume Element (RVE) is also crucial. Too small area of a non-periodic material microstructure can be misleading.

\section{Synthetic and semi-synthetic modelling}

Intrinsic property of image based FE modelling is that it is not modifiable for examples by changing the volume fractions of the material phases (e.g. wood fibers and matrix), except for small changes in the segmentation, for examples by modifying fiber thicknesses. The values describing the material properties can be changed easily, though. The synthetic tessellation based models are more suitable for predicting changes in volume fraction, particle size etc. A basic 3D tessellation routine uses a cloud of points in a volume of space, and divides the volume into sub-volumes by creating boundaries that are at equal distance from each of the closest points. By modifying the routine, it is possible to add more complicated features to the model to obtain for examples a microstructure resembling and statistically near-equivalent to that of a steel. A combination of these two methods is the semi-synthetic models, in which representative objects from an image based model are added one after another into a synthetic model in order to obtain statistically near-equivalent model, which is easy to modify for volume fraction by modifying the script creating the model. 


\section{Solving the models and analyzing the results}

Typically the resulting FE models are very large, they may contain tens of millions of elements or more. If the material model is also complicated, for examples a viscoelastic or viscoplastic model, the running of the model on a PC would take months. Utilizing high-performance computing clusters single runs usually go through in hours or days.

After solving the model the results are studied from contour plots, from XY plots of for example stress and strain histories at a node, and also statistically - by studying distributions and cumulative distributions of stresses and strains. These distributions and the contour plots together have revealed that the highest stresses in such a model often occur in very small areas and can be of one or even two magnitudes higher than the bulk stress and strain. If there are too many of these excess stress concentrations, the model has to be made again. But if there are only a few, they can often be neglected, because even a very high stress in a very small area usually cannot initiate a crack in a real material. Still, even in larger areas the stresses in the stiffer phase(s) in the microstructure tend to be much higher than that of the bulk material in a tensile test (from 3 to 5 times higher in the WPCs studied recently at VTT).

\section{Utilizing the results}

There are several ways how the results of these models are utilized. One is to give insight to the phenomena and mechanisms of deformation. More and more these models are used to predict stiffness and strength of heterogeneous and non-periodic composite materials with less experiments than what would be necessary without modelling. Also mechanisms of failure (fracture, creep) are studied with these models, especially in metals, and material microstructures chosen or even tailored for some application based on the understanding and phenomena in the microscale. This is still somewhat young viewpoint for material analysis and is developing rapidly. The results of these models are also used in prediction of friction and wear of materials under severe loadings and environmental conditions, for example wear resistant steels exhibiting Twinning Induced Plasticity (TWIP) during operation in a jaw crusher crushing rocks.

One interesting application area is predicting fatigue in metals. For this special Crystal Plasticity Finite Element (CPFE) models are used. In such a model the microstructure and crystal orientations are taken from EBSD images. The CPFE models assume that plastic deformation in crystalline material occurs along predetermined orientations along the slip systems typical for these crystals. A typical result in such a study is the distribution of the cumulative slip, which can be utilized as a measure of damage.

The application area and the customer pool of VTT's ProperTune is large and growing. Steel and material manufacturers and many machine and equipment producers are typical customers. On the composite side we have worked for examples with forest industry and companies producing components for the automotive sector. Our domestic and international collaboration network is large and contains most of the foremost research groups in the field.

In some of the high value steels of today one or more solid-to-solid transformations are utilized. For example Transformation Induced Plasticity (TRIP) and TWIP steels are 
used in mining industry, e.g. in truck loaders and excavators, these transformations are utilized to resist wear. In the future the FE modelling will be linked with the CALPHAD thermodynamics (ThermoCalc, FactSage, etc.) in order to predict which phase transformations are possible in these materials under the load and conditions given and the Phase Field method to provide physics-based prediction of associated kinetics.

An important development is linking the microstructures and strength of the material under combined loadings, linking material behavior at laboratory and actual operational conditions. This calls for a parallel experimental and modelling study - of the typical structural steels for example - in which the bulk loadings in tensile, compressive and shear tests and the probability levels of stresses and strains in the microstructure are linked together. This should first be done at room temperature and then at elevated temperatures. This is a formidable task, but the gain would be huge: The results would enable tailoring of materials to the applications and loadings, as the design engineer would have tools for taking into account the microstructure in designing the structural component of a machine or a building.

\section{ProperTune ${ }^{\mathrm{TM}}$}

Image based modelling of 3D tomography data

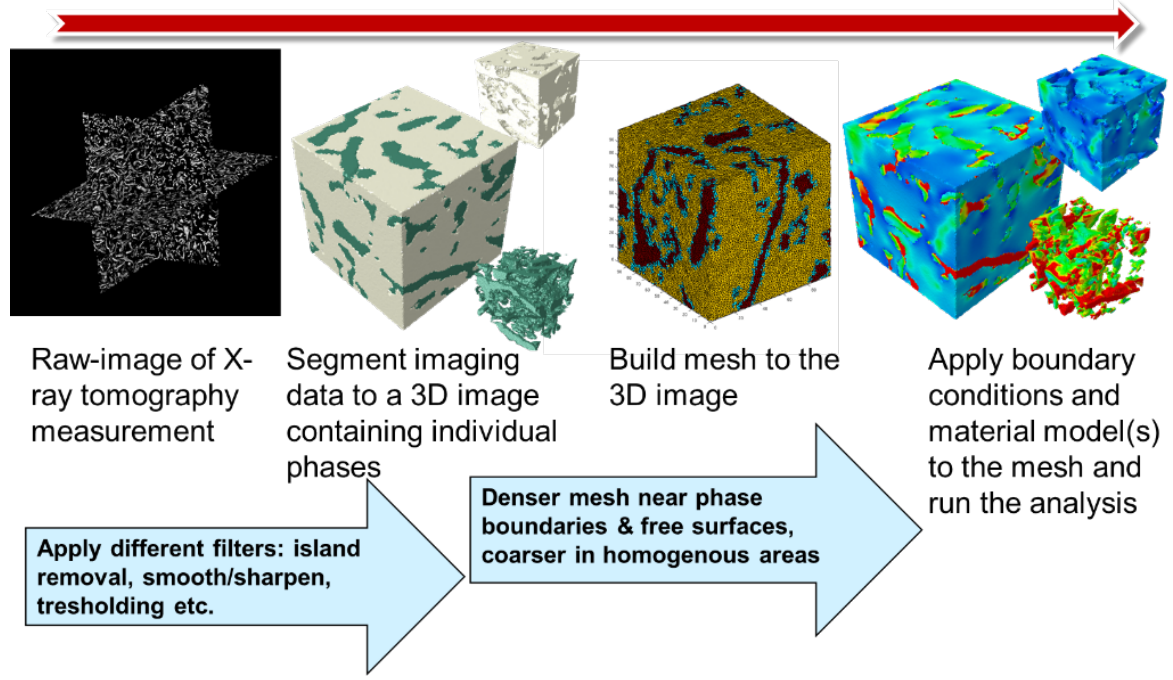

Figure 1. Workflow in image based finite element modelling.

\section{References}

[1]. M. Sippola, K. Immonen, A. Miettinen, A. Laukkanen, T. Andersson, H. Peltola, A. Harlin, K. Holmberg. Predicting stiffness and strength of birch pulp-Polylactic acid composites. Journal of Composite Materials, 50(18):2549-2563, 2016. https://doi.org/10.1177/0021998315608431

[2]. K. Holmberg, A. Laukkanen, E. Turunen, T. Laitinen. Wear resistance optimisation of composite coatings by computational microstructural modelling. 
Surface and Coatings Technology, 247:1-13, 2014.

https://doi.org/10.1016/j.surfcoat.2014.02.019

Merja Sippola, Anssi Laukkanen, Tom Andersson, Matti Lindroos

VTT Technical Research Centre of Finland

P.O Box 02044 VTT Finland

firstname.surname@vtt.fi 